\title{
Investigations on the anisotropic yield behaviour of a cohesive bulk solid
}

\author{
F. Saraber \\ Shell International Chemicals Ltd., Department CMFE, P.O. Box 162, 2501 AN The Hague (The Netherlands) \\ G. G. Enstad \\ P.wder Science and Technology Research A/S, Kjølnes Ring, N-3900 Porsgrunn (Norway)
}

and G. Haaker

University of Twente, Department of Mechanical Engineering, P.O. Box 217, 7500AE Enschede (The Netherlands)

(Received June 13, 1988; in revised form December 13, 1989)

\begin{abstract}
The theoretical treatment of the failure behaviour of bulk solids is often based on the simplifying assumption that the material acts as an isotropic continuum, regardless of the direction in which the stresses are acting. This article describes the results from measurements with both a standard Jenike shear tester and a biaxial tester which indicate that the yield behaviour of a cohesive solid can show significant anisotropic effects depending on the directions of the principal stresses at steady-state and incipient failure. Moreover, it can be concluded from those measurements that neither the flow function nor the density of a cohesive bulk solid can be regarded as an invariant with respect to stress history without taking into account possible anisotropic effects.
\end{abstract}

\section{Introduction}

Since Jenike [1] in the early sixties published his theory of the flow behaviour of bulk solids in silos, a reliable method has been avalable for the geometric design of silos. In order to measure the flow properties needed in the theory, he also introduced a modified version of a direct shear tester (known from the field of soil mechanics) and a measuring procedure suitable for cohesive bulk solids. However, to arrive at a solution within a reasonable time, many simplifying assumptions had to be made. Later on, refinements to some of these assumptions were suggested [2], whereas others up till now have remained unaltered. An example of the latter is the assumption that the powder behaves as an isotropic continuum (where the term isotropic means that the powder has the same strength regardless of the direction in which stresses are acting). However, some workers have already shown that powders are not isotropic in this respect $[3,4]$ and the main purpose of the present contribution is to make an initial evaluation of the deviations powders show from the ideal isotropic behaviour originally assumed.
The failure behaviour of a cohesive powder is generally described by so-called yield loci. A yield locus expresses the shear strength of a bulk solid as a function of the normal stress at a certain density of the bulk solid (or better still: a certain stress history during consolidation to this particular density). The flow properties, relevant for silo design, can be derived from a family of yield loci by drawing Mohr circles tangential to a yield locus, see Fig. 1. This leads, among other things, to a relation between the unconfined yield strength $f_{\mathrm{c}}$ and the major consolidating stress $\sigma_{1}$, usually called the flow function.

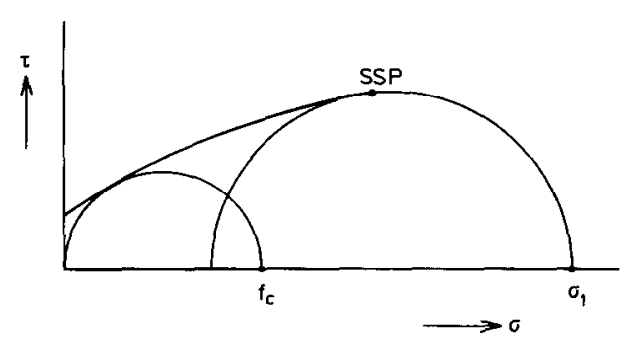

Fig. 1. Yield locus of an arbitrary bulk solid. 
In Jenike's theory, the unconfined yield strength $f_{\mathrm{c}}$ is assumed to be the strength of the material from which a possible arch or channel is formed. Obviously, such an obstruction can only be stable when the principal stress acting upon it $\left(\sigma_{1}{ }^{\prime}\right)$ is smaller than the strength of the material $f_{c}$ in the obstruction. This so called flow/no-flow criterion causes no problem when the material behaves isotropically. However, in the case of anisotropic yield behaviour, the direction of principal stresses should be taken into account. See for instance Fig. 2, which shows possible directions for the major principal stress $\sigma_{1}$ during flow in a hopper, and the major principal stress $\sigma_{1}{ }^{\prime}$ acting when an arch is formed. Normally, such an arch is unstable when $\sigma_{1}{ }^{\prime}$ is larger than $f_{c}$, resulting from the consolidation by $\sigma_{1}$. However, when the strength of the material depends on the direction of the stresses (i.e., anisotropy), one has to deal with the value of $f_{c}$ in the direction of $\sigma_{1}{ }^{\prime}$, which may deviate by an angle $\alpha$ from the direction of $\sigma_{1}$. In the case of stable arches, the directions of $\sigma_{1}$ and $\sigma_{1}{ }^{\prime}$ deviate only slightly and the influence can generally be neglected. In the case of a stable vertical channel, the deviations are more pronounced. Here (at least in Jenike's under bound approach [1]), the material in the channel wall is assumed to be consolidated by the stresses exerted by the material inside the channel. If, accordingly, failure of the empty channel is considered, a situation has to be dealt with where the inside of the channel is traction free. Hence, the stress situations of consolidation and failure are rather different in this case and a possible anisotropy should be considered.

In order to ascertain the importance of possible anisotropy, a set of experiments has been performed where the unconfined yield strength $f_{\mathrm{c}}$ is measured at different angles $\alpha$ to the consolidating stress $\sigma_{1}$.

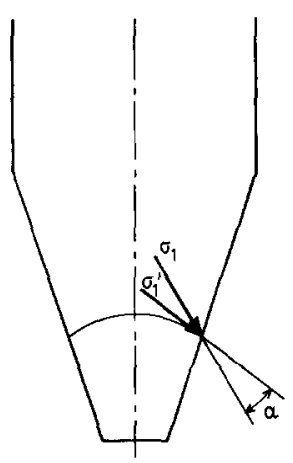

Fig. 2. Possible directions of $\sigma_{1}$ during flow and $\sigma_{1}{ }^{\prime}$ at arching in a hopper.

\section{Test equipment}

\section{Jenike shear tester}

The measurements were carried out by means of a standard Jenike shear tester and a biaxial tester with flexible boundaries (cubic plain-strain tester). The Jenike shear tester and standard measuring procedure have been amply discussed by Jenike and Schwedes $[1,4]$. Within the scope of this article only a short description is given. It is the aim of the tester to measure points of incipient failure in conjunction with steady-state failure points. Tests are performed at three stages, see Fig. 3. First the cell (with extra filling ring) is uniformly filled and the material is consolidated by twisting the cover under a vertical pressure $\sigma_{c}$. This stage should consolidate the material to a degree somewhat lower than the final density from the steady state. The filling ring and top layer of material are then removed and the sample is loaded by means of a shearing lid with a vertical pressure $\sigma_{\mathrm{pr}}$. The sample is now forced to shear by a continuous horizontal displacement $s$ of the ring over the steady base, until a steady-state failure condition is reached. This is marked by the shear stress $\tau_{\mathrm{pr}}$ reaching a constant value. The stresses $\sigma_{\mathrm{pr}}$ and $\tau_{\mathrm{pr}}$ from this steady state are indicated in Fig. 1 as the point SSP from which the major consolidating stress $\sigma_{1}$ can be derived. After reaching the steady state, the vertical stress is reduced to $\sigma_{\mathrm{s}}$ and shearing is continued until incipient failure of the sample occurs, indicated by the shear stress $\tau_{\mathrm{s}}$ passing a maximum value. The relevant stresses from
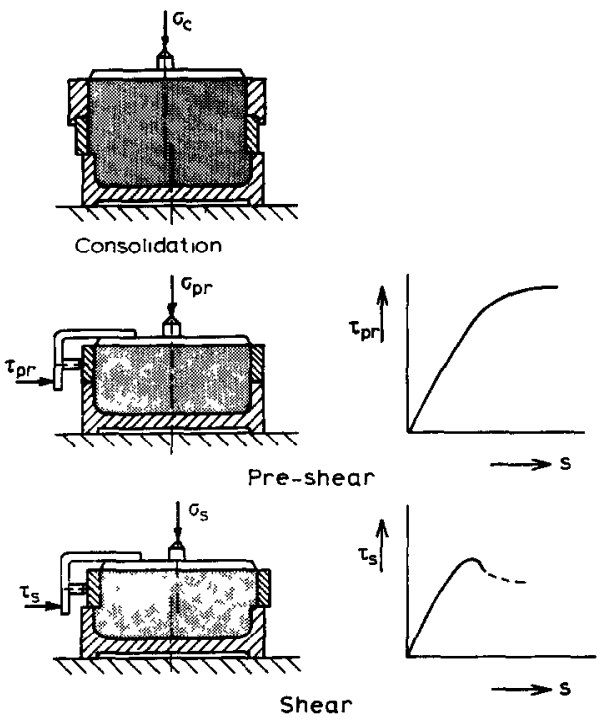

Fig. 3. Test stages during measurements with the Jenike shear tester. 
this stage lead to one point of the yield locus. In order to measure other points of the same yield locus, the whole procedure must be repeated on new samples with the same values of $\sigma_{\mathrm{c}}$ and $\sigma_{\mathrm{pr}}$ but different values of $\sigma_{s}$. Other yield loci can be measured by the same procedure but at different $\sigma_{\mathrm{pr}}$ levels.

As amply discussed in the literature [1,5], the direction of the major principal stress during the measurements is located at an angle of about $45^{\circ}+\phi_{1}$ to the vertical for both the steady-state and incipientfailure points. Although the values of the internal friction angle $\phi_{1}$ differ for both situations, these deviations in the direction of $\sigma_{1}$ are small and generally neglected. It should be noted, however, that if we wish to simulate the influence of timeconsolidation in the tests, the sample (after preshearing) is placed in a consolidating bench. There it is loaded for a prescribed interval of time with only a vertical deadweight, equivalent to the value of $\sigma_{1}$ from the steady-state consolidation. Therefore, in this case, the direction of $\sigma_{1}$ is also vertical and deviations due to a possible anisotropy might play a role.

\section{The biaxial tester}

A complete description of the biaxial tester is given in a publication by Arthur et al. [6] but Fig. 4 will give a general idea of the basic principles of the apparatus. The cubic sample of the material to be tested is enclosed in two directions by flexible membranes, filled with air at a controlled pressure. In the vertical direction, the height of the sample is kept constant by covers, greased to minimize the friction. The backing plates of the membranes can slide along grooves in the retaining guides, which themselves can be moved backwards and forwards. The exact position of the front faces of the membranes can be followed by an electrical sensing circuit between membrane and backing plate. By adjusting the air pressure and displacement of the membranes,

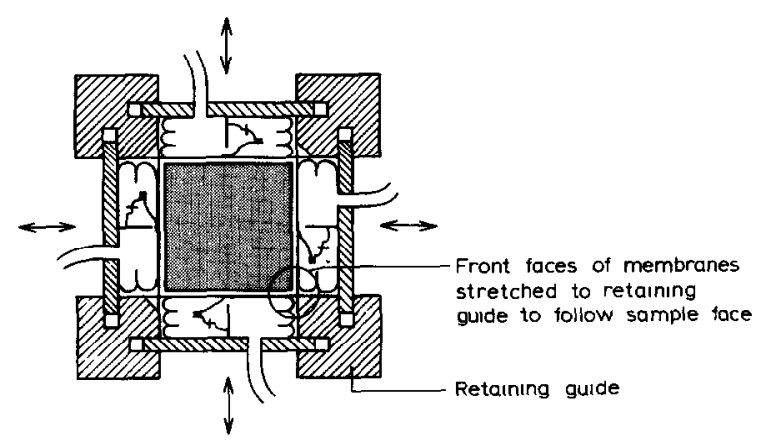

Fig. 4. Schematic section of the biaxial tester. the sample can be forced to follow a chosen deformation path, simultaneously measuring or controlling principal stresses and deformations. A comprehensive description of the measuring technique and its interpretation is published elsewhere [6].

\section{Test procedure}

\section{Jenike tester}

In ordinary tests, the deviation in the direction of the major principal stress $\sigma_{1}$ during the steady state and incipient failure, respectively, is small and generally neglected. In this article, this situation is denoted as tests with $\alpha=0$. However, in some of our tests, we deliberately created a deviation in the directions of $\sigma_{1}$, further denoted as tests with $\alpha>0$.

For $\alpha>0$, the adopted procedure is as follows:

In the normal way, the first stages of the test (filling, twisting and pre-shearing to a steady-state consolidation) are performed. Then the complete cell (ring and base) is rotated through a certain angle $\alpha$ around the vertical axis, taking care not to disturb the sample by relative movements between ring and base. In this position, the sample is brought to shear in the original direction under a lesser vertical load.

The following test program was chosen:

- Measurement of yield loci at four different steady-state stress levels with $\alpha=0$. From this family of yield loci, the flow function (i.e., the $f_{\mathrm{c}}-\sigma_{1}$ relation) can be derived.

- Measurements of yield loci at one steady state stress level for various values of $\alpha$ in the range $0^{\circ}<\alpha<180^{\circ}$. As the region for $\alpha$ up to $30^{\circ}$ is the most interesting part with regard to hopper design, a large number of measurements was chosen within this region.

\section{The biaxial tester}

Use of the biaxial tester at $\alpha=0$

With the biaxial tester, it is possible to measure points of the flow function directly instead of deriving the values from complete yield loci.

The determination of one point on the flow function can be divided into two main stages. The first one is the steady state determination that gives the $\sigma_{1} /$ $\sigma_{3}$-combination during steady state consolidation, see Fig. 5(a). The second stage is the determination of $f_{c}$ by shearing with $\sigma_{3}=0$, see Fig. $5(\mathrm{~b})$. It should be noted that the value of $f_{\mathrm{c}}$ is measured in the direction of $\sigma_{1}$ from the steady state consolidation, so that in this case $\alpha=0$. 


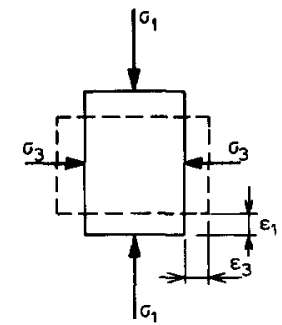

(a)

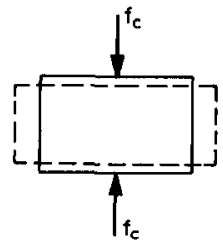

(b)
Fig. 5. Illustration of the determination of one point on the flow function with the biaxial tester, $\alpha=0$. (a), Steady state; (b), unconfined yielding.
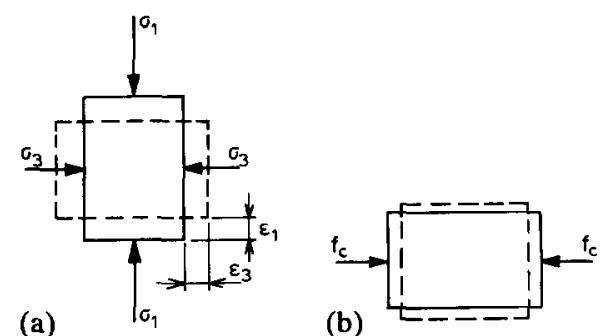

Fig. 6. Determination of one point on the flow function with the biaxial tester at $\alpha=90^{\circ}$.(a), Steady state; (b), unconfined yielding.

Use of the biaxial tester at $\alpha=90^{\circ}$

Figure 6 gives the method for measuring a point of the flow function for $\alpha=90^{\circ}$. It can be seen that after steady-state consolidation (Fig. 6(a)), the unconfined yield stress $f_{\mathrm{c}}$ is measured in the direction of the former minor principal stress $\sigma_{3}$ (Fig. 6(b)).

With the biaxial tester, it is only possible to measure at $\alpha=90^{\circ}$ and $\alpha=0^{\circ}$, in contrast to the tests with the Jenike tester, where measurements can be made at any angle between 0 and $180^{\circ}$.

It should be noted that, strictly speaking, the meaning of the angle $\alpha$ is different for the two testers. In the biaxial tester, the principal stress co-ordinate system remains the same, while with the Jenike tester this co-ordinate system is rotated (relative to the sample) around the vertical axis. However, applying an angle $\alpha$ means for both testers that the direction of the major principal stress at steady state is different from the one at failure.

Using the biaxial tester, five points of the flow function were measured with both $\alpha=0^{\circ}$ and $\alpha=90^{\circ}$. The steady-state consolidation was in all cases performed by the following procedure:

(i) the initial samples of $118 \mathrm{~mm} \times 118 \mathrm{~mm} \times 102$ $\mathrm{mm}$ were isostatically compacted to $\sigma_{\mathrm{o}}$,

(ii) from there on, the mean stress $\sigma_{\mathrm{m}}$ $\left(\sigma_{\mathrm{m}}=\left(\sigma_{1}+\sigma_{3}\right) / 2\right)$ was kept constant while $\sigma_{1}$ was increased and $\sigma_{3}$ is decreased until a steady-state deformation was obtained,

(iii) the values for $\sigma_{\circ}$ were chosen at: $1,3,6,9$ and $12 \mathrm{kPa}$.

\section{Conditions and results}

\section{Conditions}

All the tests were performed with finely ground natural limestone, with $95 \%$ having a diameter less than $10 \mu \mathrm{m}$.

The temperature during the tests was $20 \pm 2{ }^{\circ} \mathrm{C}$ and the relative humidity ranged from 26 to $48 \%$. The moisture content was not checked, but can be assumed to have been constant throughout the tests.

\section{Jenike tests}

Before we can discuss the results of the Jenike tests with $\alpha=0$ and $\alpha>0$, some explanation is needed.

In the experiments with $\alpha>0$, a phenomenon occurred which we have called a renewed steady state. This behaviour will be explained with reference to Figs. 7 and 8.

In the left-hand part of Fig. 7, the stress-strain curves for two normal tests with $\alpha=0$ are shown. In this case, the material is consolidated to steady state (curves a) and then sheared at a lower normal stress leading to a peak shear-stress value at incipient failure (curves b), because at this lower stress level the material behaves as an over-consolidated sample. These peak values lead to points of the yield locus as indicated in the right-hand part of the figure. The steady-state shear-stress values enable us to define the end point of the yield locus, indicated here as SSP.

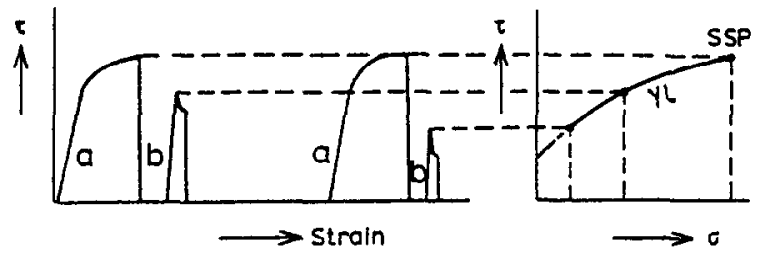

Fig. 7. Stress-strain curves and construction of the yield locus $(\mathrm{yL})$ at $\alpha=0$. SSP $=$ Steady State Point.

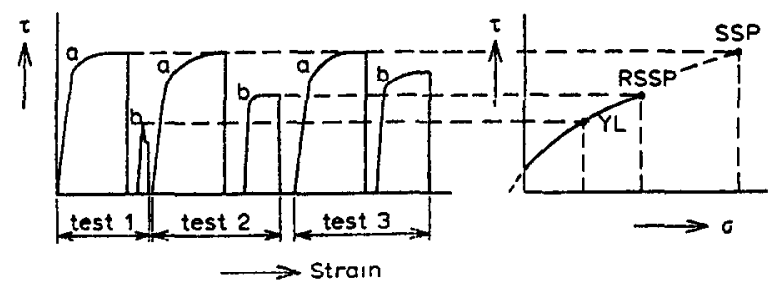

Fig. 8. Stress-strain curves and construction of the yield locus for $\alpha>0$. RSSP $=$ Renewed Steady State Point. 
For $\alpha>0$, however, the situation may be different. In Fig. 8, the results of three tests as observed during the measurements are shown. The steady-state results for all three tests are the same (curves a). At low normal-stress levels for incipient failure, the sample still behaves as an over-consolidated sample (see test 1 , curve b), which means that the shear stress shows a peak, as is the case for $\alpha=0$. At higher normal-stress levels, but still beneath the steadystate stress level, the material may behave as if it was under-consolidated (see test 3 , curve b), indicated by an increase of the $\tau$-value. Somewhere between those two cases, a normal-stress level can be found where the material behaves as a critically consolidated sample (test 2 , curve b), with the value of $\tau$ remaining constant. This indicates a renewed steady-state consolidation although at a lower stress level than the original steady state (see curve a). Based on this renewed steady-state concept, a yield locus and the renewed steady-state point (RSSP) can be constructed, as shown in the right-hand part of Fig. 8.

In all our measurements with various values of $\alpha>0$, this renewed steady-state behaviour occurred at a certain normal-stress level.

The derivation of the $f_{c^{-}}$and $\sigma_{1}$-values in the normal case and when adopting the renewed steadystate concept are shown in Fig. 9. This figure shows a yield locus for $\alpha=0$ and the related steady-state point (SSP) leading to one point $\left(f_{\mathrm{c}}-\sigma_{1}\right)$ on the flow function.

When $\alpha>0$, a somewhat lower yield locus is obtained, leading to a smaller value of $f_{c}$. This value can be related to the original value of $\sigma_{1}$ or to the value $\sigma_{1 \mathrm{r}}$ which can be derived using. the renewed steady-state point (RSSP).

Results derived from the Jenike tests with $\alpha=0$ and $\alpha>0$ are given in Table A.1 (see Appendix) and as a flow function in Fig. 10. When $\alpha>0$, the $f_{c}$-values are related to $\sigma_{1}$ as well as $\sigma_{1 \mathrm{r}}$, according to the renewed steady-state concept.

The unconfined yield strength $f_{\mathrm{c}}$ as a function of $\alpha$ is shown in Fig. 11.

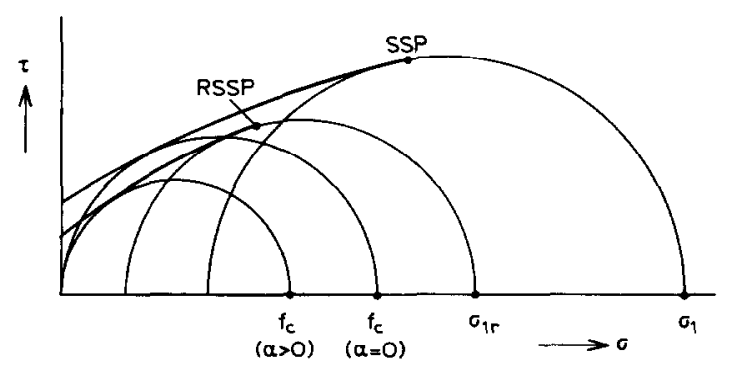

Fig. 9. Derivation of a point of the flow function for $\alpha=0$ and $\alpha>0$.

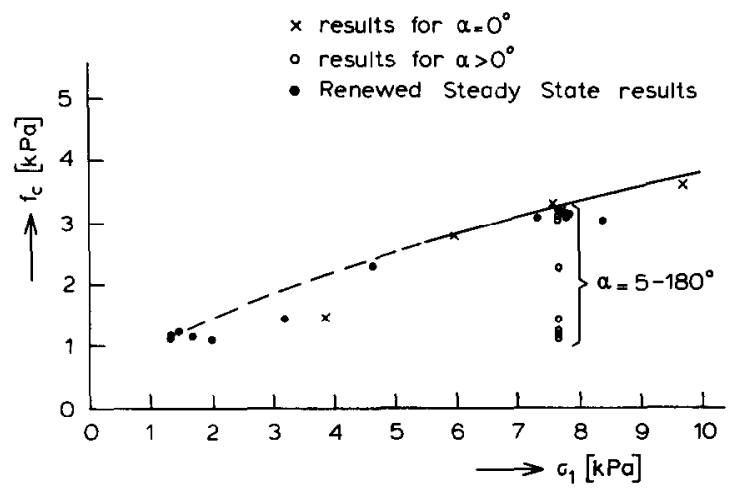

Fig. 10. The flow function derived from Jenike shear tests for $\alpha=0$ and $\alpha>0$.

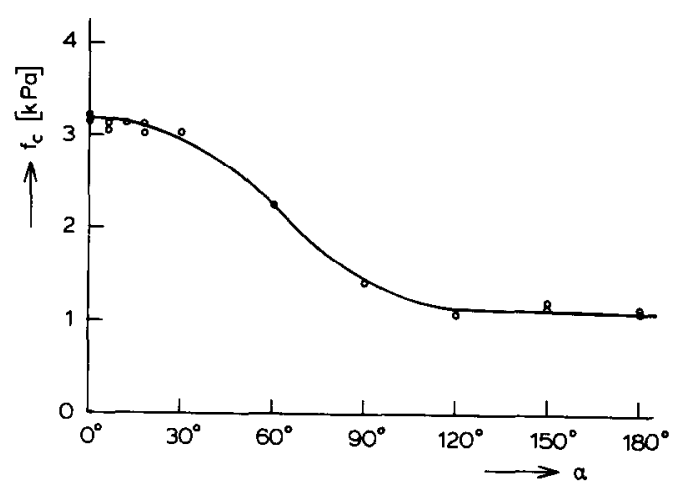

Fig. 11. The unconfined yield strength $f_{\mathrm{c}}$ as a function of $\alpha$ derived from Jenike results, with $\sigma_{1} \cong 7.7 \mathrm{kPa}$.

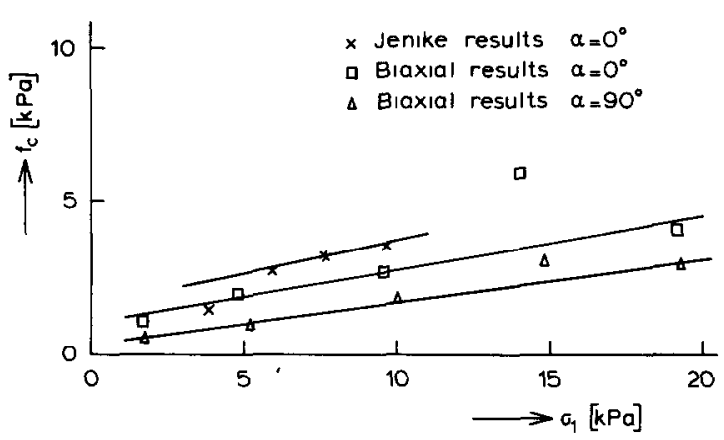

Fig. 12. Flow functions as derived from Jenike and biaxial tests.

\section{Biaxial tests}

The unconfined yield strength was only measured directly in the biaxial tester, omitting the determination of complete yield loci. It was for this reason that the phenomenon of the renewed steady state could not be investigated in those tests.

Numerical results for $\alpha=0$ and $\alpha>0$ are given in Table A.2 (see Appendix). The flow functions are 
plotted in Fig. 12 for comparison with the results from the Jenike tester.

\section{Discussion and comparison of results}

\section{Discussion of the actual measurements}

In this section, a general description of the uncertainties in determining the yield loci and flow functions will be discussed.

\section{Jenike test results $\alpha=0-$ yield loci $1-5$}

No problems occurred in determining the yield loci. The only point of discussion is the lowest point of the flow function with $\sigma_{1}=3.8 \mathrm{kPa}$ and $f_{\mathrm{c}}=1.44$ $\mathrm{kPa}$, which falls far below the trend of the higher points. This is most probably due to the high scatter encountered in this low-stress region. For that reason, the point is neglected when plotting the flow function.

\section{Jenike tests results $5 \leqslant \alpha \leqslant 180$ - yield loci $6-19$}

The determination of the renewed steady state was sometimes a problem in this region. Either an over-consolidated or an under-consolidated behaviour was observed. But efforts were made to determine the renewed steady-state point as reliably as possible which, in some cases, meant repeating the tests.

Another problem was that with $\alpha>120^{\circ}$ difficulties occurred in determining the yield loci. This is because the end points and starting points that define the region of valid measurements are so close together that it was hardly possible to measure within this region and to draw the actual yield locus afterwards. In this case too, efforts were made to make the results as reliable as possible. That is why two yield loci were determined for $\alpha=150^{\circ}$ and $180^{\circ}$.

\section{Biaxial test results}

It could be concluded from the stress-strain curves and the strain-rate data (not given in this publication) that the steady-state data were determined reliably. The criterion that steady state occurs is a continuous deformation of the sample under constant volume. This means in the practical situation that the continuous movement is only limited by the dimensions of the cell. However, to measure the $f_{\mathrm{c}}$-value, both the major and the minor principal stress must be altered in order to stop this continuous movement. This was done by increasing the minor and decreasing the major principal stress.

At high stresses, however, the problem arose that the rate of outward motion was larger then the rate of inward motion. After trying to stop the continuous deformation, the outward motion stopped but the inward motion still went on in order to adjust itself to the total outward motion. In order not to run out of strain (limitations of the cell dimensions), the inward motion had to be stopped twice manually. Although in those cases the bags (see Fig. 4) were not exactly plane, the $f_{\mathrm{c}}$-value was determined. This, of course, influenced the strain rate but probably also influenced the $f_{\mathrm{c}}$-value, although to what extent is difficult to tell.

It can be observed in Fig. 12 that the point on the flow function $(\alpha=0)$ with $\sigma_{1}=14 \mathrm{kPa}$ and $f_{\mathrm{c}}=5.9$ $\mathrm{kPa}$ does not fall into line with other points. The explanation may be that before determining the $f_{c}$ value and after steady state, the $\sigma_{3}$-bags were not retracted enough, which causes the $f_{\mathrm{c}}$-value to end up higher than it should be.

\section{Comparison of results}

In Fig. 11, the unconfined yield strength $f_{c}$ is given as a function of $\alpha$, based on Jenike shear tests. It is seen from this curve that $f_{\mathrm{c}}$ has a maximum value for $\alpha=0$, but for $\alpha<30^{\circ}$ only a small decrease up to about $5 \%$ in the $f_{\mathrm{c}}$-value occurs. In the case of $\alpha>30^{\circ}$, a considerable drop in the $f_{\mathrm{c}}$-value can be seen, whereas from $\alpha=120^{\circ}$ it becomes almost constant. In the last region, however, the uncertainty in the determination of the yield locus must be kept in mind.

A comparable plot is not available for the biaxial results, because only tests with $\alpha=0^{\circ}$ and $\alpha=90^{\circ}$ could be performed. It can be seen from the values in Table A2 that a considerable drop in the $f_{\mathrm{c}}$-values also occurs for $\alpha=90^{\circ}$.

A straightforward comparison of the drop in the $f_{\mathrm{c}}$-values for both testers is hardly possible because of the different meaning of the angle of rotation $\alpha$ and the variation in $\sigma_{1}$-values.

A better comparison can be found if we look at the flow functions as given in Fig. 12. The flow functions for $\alpha=0^{\circ}$ show that the biaxial tester leads to a significantly lower flow function. This is in line with previous findings of over design in the standard Jenike method for determining critical outlet dimensions of mass-flow hoppers [7].

More interesting, in the view of anisotropy, is the comparison of the flow functions from the biaxial tester for $\alpha=0^{\circ}$ and $\alpha=90^{\circ}$, respectively, as shown in Fig. 12. It is obvious that the unconfined yield strength is much lower for $\alpha=90^{\circ}$, which indicates significant direction-dependent behaviour.

The same tendency can be found from the results of the Jenike tests as shown in Fig. 10. Due to the restrictions on the number of measurements in this case, only the influence of $\alpha$ on one point of the flow function can be shown, again indicating significant anisotropic behaviour. 
However, it is interesting to see how the flow function for $\alpha>0^{\circ}$ behaves if the idea of the renewed steady state is adopted: taking the $f_{\mathrm{c}}-\sigma_{1 \mathrm{r}}$ relation, as is also shown in Fig. 10. It is clear that apart from some scatter this result is close to the flow function for $\alpha=0$. This might give the impression that the flow function is an invariant for a given powder and is not influenced by the stress history of the powder. One must consider, however, that the concept of a renewed steady state implies that the stress history has the same influence on both the renewed steady-state and the incipient failure points. Therefore, in fact, the influence of the stress history is eliminated.

Harder and Schwedes [8] have published some of their measurements with a True Biaxial Tester (TBT). They concluded from their results that the flow function is invariant with regard to the stress history. However, in their measurements they started with samples which were consolidated with different stress histories, but from there on they determined both steady-state and incipient failure points. This implies that they used a procedure comparable to our renewed steady-state concept.

In Fig. 13, the bulk densities measured in both testers are compared, showing that the biaxial tester leads to smaller densities than the Jenike tester. This is in line with the results from Harder and Schwedes [9] and also with the measured flow functions for $\alpha=0^{\circ}$ as compared in Fig. 12. It should be kept in mind, however, that the densities in the biaxial tester are more homogeneous and are measured directly, whereas the density in the failure zone of the Jenike shear tester can deviate from the measured mean density of the whole sample.

More important, however, is the role of the density $\rho_{\mathrm{b}}$ if we compare the results for $\alpha=0^{\circ}$ and $\alpha>0^{\circ}$. These figures are giveri in Tables A.1 and A.2, showing

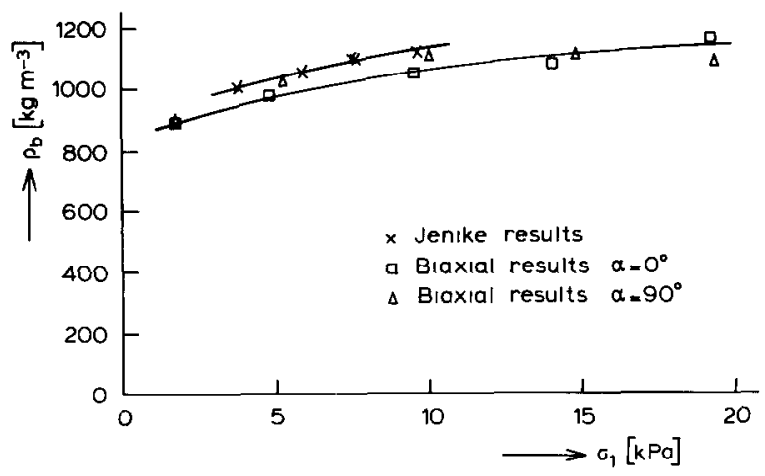

Fig. 13. The density $\rho_{\mathrm{b}}$ as a function of $\sigma_{1}$ derived from Jenike and biaxial tests. that samples of approximately the same density can lead to a large variety of $f_{\mathrm{c}}$-values, depending on the rotation angle $\alpha$. Both testers lead to the same results in this respect. This means that the density is not a unique parameter of a bulk solid with regard to the yield behaviour, but that the direction of principal stresses has to be taken into account as well.

\section{Conclusions}

Although measurements were performed on one material only, the following conclusions can be drawn from our results:

(1) The yield behaviour of a cohesive solid can show significant anisotropic effects when the directions of the principal stresses at steady state and incipient failure are different.

(2) Theoretically, this anisotropic behaviour influences the determination of the critical outlet width of a silo according to the Jenike method. However, from our practical results, it is obvious that this influence is negligible for mass-flow silos if timeconsolidation is excluded. For funnel-flow silos, the importance of anisotropy may be considerable.

(3) The flow function of a cohesive bulk solid seems to be invariant with respect to stress history, provided that the directions of the major principal stresses during steady state and incipient failure are the same. This can be achieved either directly by the measuring technique or by applying a concept similar to our renewed steady state.

(4) The density is not a unique parameter of a bulk solid with regard to yield behaviour. This implies that the results from testers based on different testing principles cannot be compared on the basis of density without taking into account possible anisotropic effects.

\section{Acknowledgements}

The authors are grateful to Dr Jenike, who suggested this investigation during stimulating discussions with one of them. The work was funded by the Royal Norwegian Council for Scientific and Industrial Research, and the measurements were performed at the Christian Michelsen Institute in Bergen, Norway.

\section{List of symbols}

$f_{\mathrm{c}}$ RSSP renewed steady-state yield point 
SSP steady-state yield point

$\alpha$ angle that might occur between the direction of the major principal stress during steady state shear, and during subsequent initial (or unconfined) yield; in the measurements deliberately attained by rotation of the sample or the principal stresses

$\rho_{\mathrm{b}} \quad$ bulk density, $\mathrm{kg} \mathrm{m}^{-3}$

$\sigma_{1} \quad$ major principal stress at steady state, $\mathrm{N} \mathrm{m}^{-2}$

$\sigma_{1}{ }^{\prime} \quad$ major principal stress at failure, $\mathrm{N} \mathrm{m}^{-2}$

$\sigma_{3} \quad$ minor principal stress, $\mathrm{N} \mathrm{m}^{-2}$

$\sigma_{0} \quad$ isostatic compaction stress, $\mathrm{N} \mathrm{m}^{-2}$

$\sigma_{\mathrm{m}} \quad$ mean principal stress, $\sigma_{\mathrm{m}}=\left(\sigma_{1}+\sigma_{3}\right) / 2$, $\mathrm{N} \mathrm{m}^{-2}$

$\sigma_{\mathrm{lr}} \quad$ major principal stress at renewed steady state, $\mathrm{N} \mathrm{m}^{-2}$

normal vertical stress at pre-shear, $\mathrm{N} \mathrm{m}^{-2}$

$\sigma_{\mathrm{pr}}$

$\sigma_{\mathrm{s}}$

$\tau$

$\tau_{\mathrm{pr}}$

$\tau_{\mathrm{s}}$

$\phi_{1}$

\section{Appendix}

Complete numerical results of all measurements with the Jenike and biaxial testers are given in Tables A.1 and A.2

TABLE A.1. $\sigma_{1^{-}}, f_{c^{-}}$and $\rho_{\mathrm{b}}$-values determined by means of the Jenike tester

\begin{tabular}{rrllll}
\hline $\begin{array}{r}\text { YL } \\
\text { No. }\end{array}$ & $\begin{array}{l}\alpha \\
\left({ }^{\circ}\right)\end{array}$ & $\begin{array}{l}\sigma_{1} \\
(\mathrm{kPa})\end{array}$ & $\begin{array}{l}\sigma_{\mathrm{lr}} \\
(\mathrm{kPa})\end{array}$ & $\begin{array}{l}f_{\mathrm{c}} \\
(\mathrm{kPa})\end{array}$ & $\begin{array}{l}\rho_{\mathrm{b}} \\
\left(\mathrm{kg} \mathrm{m}^{-3}\right)\end{array}$ \\
\hline 1 & 0 & 3.82 & & 1.44 & 1005 \\
2 & 0 & 5.92 & & 2.76 & 1052 \\
3 & 0 & 7.65 & & 3.16 & 1097 \\
4 & 0 & 7.53 & & 3.26 & 1093 \\
5 & 0 & 9.63 & & 3.57 & 1114 \\
6 & 5 & 7.7 & 7.78 & 3.12 & 1096 \\
7 & 5 & 7.7 & 7.69 & 3.05 & 1104 \\
8 & 10 & 7.7 & 7.56 & 3.13 & 1097 \\
9 & 15 & 7.7 & 7.74 & 3.12 & 1109 \\
10 & 15 & 7.7 & 8.35 & 3.01 & 1092 \\
11 & 30 & 7.7 & 7.27 & 3.04 & 1097 \\
12 & 60 & 7.7 & 4.58 & 2.26 & 1098 \\
13 & 90 & 7.7 & 3.15 & 1.41 & 1095 \\
14 & 120 & 7.7 & 1.96 & 1.09 & 1096 \\
15 & 150 & 7.7 & 1.42 & 1.23 & 1099 \\
16 & 150 & 7.7 & 1.66 & 1.16 & 1098 \\
17 & 180 & 7.7 & 1.30 & 1.16 & 1102 \\
18 & 180 & 7.7 & 1.30 & 1.10 & 1107 \\
\hline
\end{tabular}

TABLE A.2. $\sigma_{1}{ }^{-}, f_{c^{-}}$and $\rho_{\mathrm{b}}$-values determined by means of the biaxial tester

\begin{tabular}{llll}
\hline $\begin{array}{l}\alpha \\
\left({ }^{\circ}\right)\end{array}$ & $\begin{array}{l}\sigma_{1} \\
(\mathrm{kPa})\end{array}$ & $\begin{array}{l}f_{\mathrm{c}} \\
(\mathrm{kPa})\end{array}$ & $\begin{array}{l}\rho_{0} \\
\left(\mathrm{~kg} \mathrm{~m}^{-3}\right)\end{array}$ \\
\hline 0 & 1.73 & 1.10 & 890 \\
0 & 4.81 & 2.04 & 980 \\
0 & 9.52 & 2.75 & 1050 \\
0 & 13.99 & 5.92 & 1080 \\
0 & 19.14 & 4.07 & 1160 \\
90 & 1.77 & 0.53 & 900 \\
90 & 5.20 & 0.98 & 1020 \\
90 & 10.03 & 1.79 & 1100 \\
90 & 14.78 & 3.03 & 1100 \\
90 & 19.27 & 2.98 & 1080 \\
\hline
\end{tabular}

1 A. W. Jenike, Utah Exp. Bull. 123, 1970.

2 G. G. Enstad, Ph. D. Dissertation, Christian Michelsen Institute, Bergen, Norway (1981).

3 O. Molerus, Fluid-Feststoff Stromungen, Springer, Berlin, 1982.

4 J. R. F. Arthur, M. A. Koenders and R. K. S. Wong, Acta Mechanica, 64 (1986) 19.

5 J. Schwedes, Ph. D. Dissertation, Univ. Karlsruhe, 1971.

6 J. R. F. Arthur, T. Dunstan and G. G. Enstad, Int.

J. Bulk Solids Storage in Silos, 2 (1985) 7.

7 G. G. Enstad, Chem. Eng. Sci., 30 (1975) 1273.

8 J. Hardner and J. Schwedes, Proc. 1st World Congr. Part. Technol., Numberg, Part III (1986) 143. 